\title{
Aplicação Prática de IoT e Computação Móvel no Controle de Sistemas de Ar Condicionados
}

\author{
Renan Amorim Pacheco ${ }^{1}$, Amanda Beatriz C. dos Santos ${ }^{1}$, Rafael M. da Cruz ${ }^{1}$, \\ Leonardo H. Gonsioroski ${ }^{1}$, Jairon Batista Viana ${ }^{1}$
}

${ }^{1}$ Departamento de Engenharia da Computação - Universidade Estadual do Maranhão

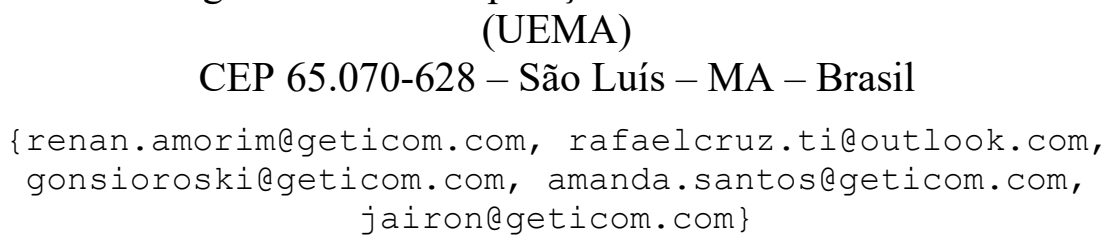

\begin{abstract}
This article presents the development of a device capable of connecting to the internet and, through it, controlling an air conditioning unit through a supervisory system accessible from any computer or smartphone. The development of the hardware of the device will be presented, which involves the creation of the circuit diagram and the printed circuit board, the development of the firmware embedded in the device and the development of the control software, using the Microsoft .NET framework. Product efficiency tests were carried out on the control of two air conditioning units. The main result of the work is understood as the materialization of a device that is simple to handle and capable of significantly reducing energy consumption expenditures, both in the control of a residential air conditioning system and of more complex business systems.
\end{abstract}

Resumo. Este artigo apresenta o desenvolvimento de um dispositivo capaz de se conectar à internet e por meio dela controlar um aparelho de ar condicionado por um sistema supervisório acessivel de qualquer computador ou smartphone. Será apresentado o desenvolvimento do hardware do dispositivo, que envolve a criação do diagrama do circuito e da placa de circuito impresso, o desenvolvimento do firmware embarcado no dispositivo e o desenvolvimento do software de controle, utilizando o framework. NET da Microsoft. Testes de eficiência do produto foram realizados no controle de dois aparelhos de ar condicionado. Entende-se como principal resultado do trabalho a materialização de dispositivo de simples manuseio e capaz de reduzir significativamente os gastos com consumo de energia tanto no controle de um sistema de climatização residencial, quanto de sistemas mais complexos empresariais.

\section{Introdução}

A concepção das aplicações de IoT (Internet of Things) é conectar qualquer dispositivo à Internet (celulares, cafeteiras, máquinas de lavar, fones de ouvido, carros, sensores inteligentes e etc) interligando esses dispositivos às pessoas e/ou outros dispositivos. $\mathrm{O}$ avanço de tecnologias de comunicações sem fio tornou possível o crescimento das aplicações de IoT com soluções inteligentes nas mais diversas áreas (social, saúde, 
transporte, segurança pública, indústria, etc), através da convergência dos mundos físico, digital e virtual. Nos últimos anos, uma grande quantidade de soluções já vem sendo desenvolvida utilizando os conceitos de IoT [Rodrigues 2018], e existe uma expectativa de que no ano de 2022 mais de 12 bilhões de dispositivos já estejam conectados à internet, é o que aponta o último relatório da Cisco Mobile VNI [Cisco 2019].

Muitas aplicações de IoT são voltadas para a concepção de Smart Buildings (Edifícios Inteligentes) e de Smart Energy (Energia Inteligente) que são comumente aplicados de forma integrada. Dentro desse contexto, diversos estudos de sistemas automatizados de ar condicionado vêm sendo realizados, pois os sistemas de refrigeração em geral representam uma grande parcela do consumo de energia. Em [Huang, Khajepour, Ding, Bagheri, and Bahrami] os autores desenvolvem um sistema baseado em um controlador de modo deslizante utilizado para rastrear o ponto ideal temperatura do ambiente e regular de forma correta o ar condicionado para maior eficiência energética. Em [Yan, Xia, Xu and Deng] e [Omer and Muhammad] os autores usam Lógica Fuzzy para desenvolver sistemas de controle de temperatura e umidade para sistemas de refrigeração ambiente.

De forma alinhada a essas tendências e voltado a uma aplicação prática, foi desenvolvido um sistema inteligente de ar condicionado baseado em BAS (Building Automation System) [Guo, Zhou e Cui 2005]. O hardware e o software desenvolvidos são apresentados. A tecnologia que permite o gerenciamento de aparelhos de ar condicionado é composta por um dispositivo e um sistema de controle. O dispositivo é desenvolvido com um receptor de infravermelho que interpreta as informações enviadas pelo controle remoto ou pelo sistema de controle. Para aproveitar as funcionalidades é necessário ter acesso a internet no local onde os dispositivos serão instalados, bem como no aparelho que está sendo utilizado para executar o sistema de controle. Algumas das funcionalidades do sistema são:

- Ligar, desligar e inserir a temperatura desejada;

- Definir limite de temperatura;

- Programar funcionamento via timer;

- Monitorar o funcionamento de aparelhos de quaisquer marcas.

\section{Desenvolvimento do Hardware do Dispositivo}

O modelo proposto apresenta o desenvolvimento de um dispositivo (hardware) que possibilita o controle de um aparelho de ar-condicionado por meio de um software instalado em um computador conectado à internet, como ilustrado na Figura 1. O dispositivo é composto por um módulo $W i-f i$, que é responsável pela Conexão do dispositivo a internet, um LED transmissor infravermelho, que envia os comandos para o ar-condicionado, e um microcontrolador com o software embarcado, que é responsável por interpretar os comandos enviados pelo software de controle instalado no computador. Todo esse processo acontece na camada física e de enlace do modelo OSI.

O desenvolvimento do protótipo passou por 5 etapas, as três primeiras referentes à parte física e as duas últimas, à parte lógica. A primeira etapa, consistiu em desenhar o diagrama do circuito eletrônico através do software gratuito EAGLE versão 6.5. 


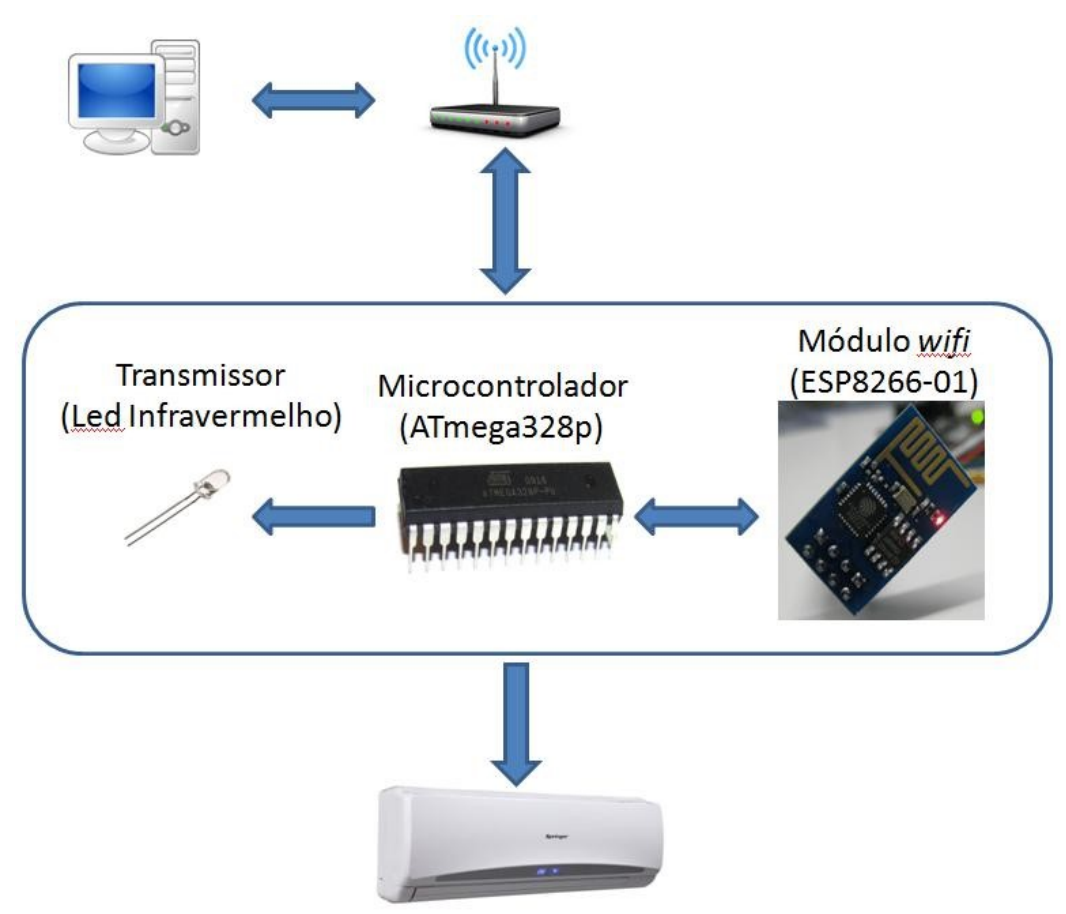

Figura 1: Sistema de Controle de Ar condicionado.

A segunda, foi o desenvolvimento do diagrama da placa de circuito impresso juntamente com o roteamento de trilha e a terceira etapa, consistiu na montagem da placa do circuito, cujo processo, resume-se em imprimir o desenho das trilhas espelhado (invertido horizontalmente) em um papel especial utilizando uma impressora.
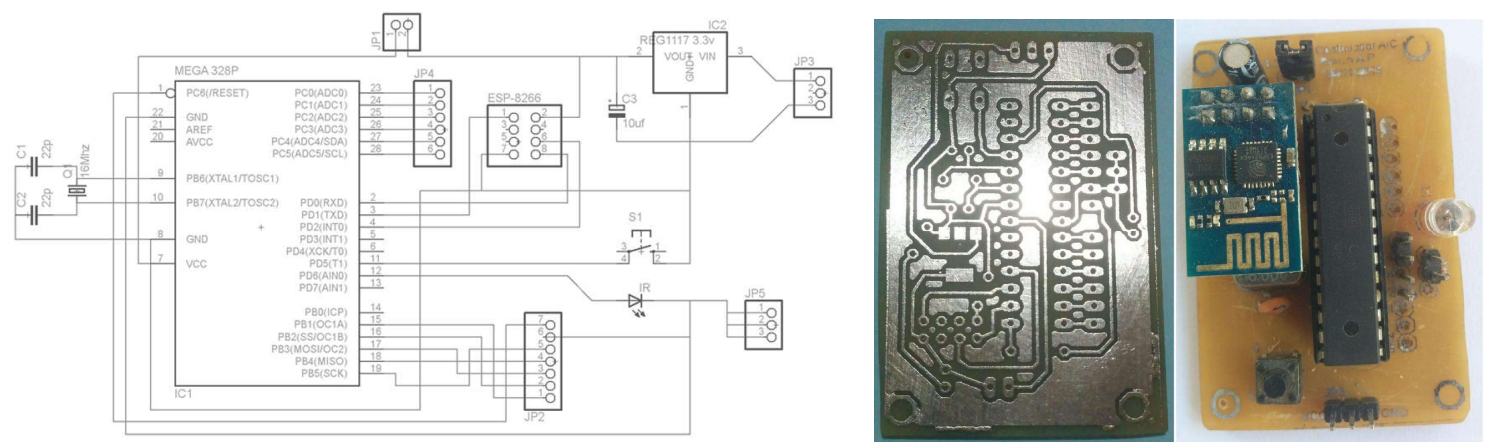

Figura 2: Diagrama do circuito, Trilhas de cobre e o Circuito Montado.

\section{Desenvolvimento do Software Embarcado}

Para o desenvolvimento do software embarcado (firmware), utilizou-se o Atmel Studio [Microchip 2018] que é um ambiente de desenvolvimento voltado para depuração e construção de aplicações para microcontroladores da família AVR. Nele as aplicações podem ser escritas em $\mathrm{C} / \mathrm{C}++$ ou em linguagem Assembly. O firmware desenvolvido contém funções que permitem a comunicação entre o microcontrolador ATmega328p e o módulo ESP8266-01. Por meio dessa comunicação é feita a configuração do módulo (configuração da rede wi-fi, modo de funcionamento), bem como, enviar dados ou recebê-los do servidor. Também foram elaboradas funções para o envio de sinais via 
infravermelho, desta maneira é possível controlar o ar-condicionado. Observa-se na Figura 3, que a composição do código fonte é formada por 3 arquivos. $\mathrm{O}$ arquivo principal "main.c", o arquivo "UART.c" e o arquivo "IR.c".

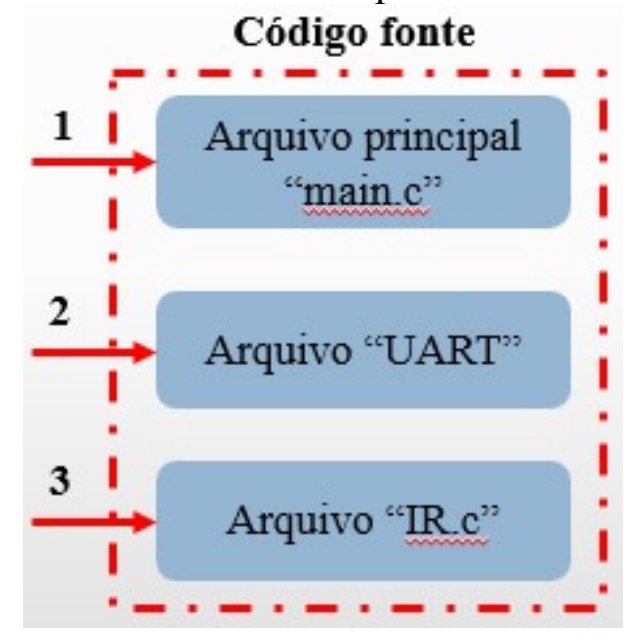

Figura 3: Arquivos do código fonte do software embarcado.

O arquivo "main.c" (1) contém as principais funções para a comunicação com o servidor e outras configurações do dispositivo, como a função de configuração do wifi e a do controle do ar-condicionado. A função Start é a primeira função executada quando o sistema é energizado. Como o ESP8266 demora alguns milissegundos até estar pronto, é preciso aguardar até que ele envie uma mensagem informando que está pronto para operar (Ready). Em seguida, é verificado se o botão de configuração está pressionado e, caso positivo, a função ConfigMode será executada. Porém, se o botão não estiver pressionado a função de conexão ESP_Connect será executada.

O arquivo "UART.c" (2) é responsável pelo tratamento da comunicação UART, ou seja, tem as rotinas para a configuração, transmissão e leitura de dados seriais que utilizam o padrão UART. É através do arquivo "UART.c" (2) que o hardware interno do microcontrolador ATmega328p responsável pela comunicação serial, é inicializado, assim como, o envio e recebimento de dados e comandos do módulo ESP8266-01.

O arquivo "UART.c" tem as rotinas para configuração, transmissão e leitura de dados seriais que utilizam o padrão UART. A função UART_init faz a inicialização do hardware interno do microcontrolador ATmega328p responsável pela comunicação serial. Essa função configura a taxa de transmissão a $115200 \mathrm{kbps}$, utilizando 8 bits de dados, 1 stop bit sem paridade. As funções UART_Write e UART_Read respectivamente enviam e recebem bytes, ou seja, por meio dessas funções é possível enviar e receber dados e comandos do módulo ESP8266-01.

Para que seja possível a conexão com o servidor, é preciso saber qual é o endereço IP na rede. Por esse motivo a função de configuração ConfigMode foi criada. Assim, permitindo salvar o endereço IP do servidor na memória do microcontrolador. Para isso, a função ativa o ESP8266 no modo AP (Access Point) com o identificador da rede definido como "ConfigMode" e senha "87654321". Deste modo, é possível enviar os parâmetros de IP do servidor utilizando um computador conectado a essa rede. Os parâmetros de configuração devem ser enviados seguindo um padrão. Como exemplo, para configurar o endereço IP "10.0.0.20” será enviada a seguinte mensagem: 


$$
\text { \#CFGA:AT+CIPSTART=|"TCP|",|"10|",80|r|n. }
$$

Outro parâmetro importante é a identificação do dispositivo. Podendo conter até oito caracteres, ela identifica em qual ambiente o dispositivo está instalado. Como exemplo, para configurar um dispositivo no Laboratório de Automação poderia ser enviada a seguinte mensagem:

$$
\text { \#CFGB:\$LABAUTO|r|n }
$$

O arquivo "IR.c" (3) contém as funções para envio do sinal via infravermelho e também é responsável pelo tratamento dos dados que serão enviados via infravermelho. Ele contém a função IR_init que configura o pino conectado ao LED como porta de saída. A função IR_send recebe um array com a sequência de dados binários a serem enviados por infravermelho. Para isso, essa função verifica cada byte a ser enviado e, quando valor binário ' 1 ', faz a chamada da função IR_Hi para enviar nível lógico alto ou, quando valor binário ' 0 ', faz a chamada da função IR_Lo para enviar nível lógico baixo. Desta forma, a função IR_send é chamada quando é preciso enviar um comando para o ar-condicionado, como o comando de ligar.

A função ESP_Connect é responsável por enviar os comandos de conexão a uma rede wifi para o módulo ESP8266-01. Ela inicialmente aguarda alguns segundos até que o ESP8266-01 esteja estável, em seguida ela inicia as tentativas de conexão em uma rede wifi.

\section{Desenvolvimento do Software de Controle - Sistema Supervisório}

O desenvolvimento do software de controle foi feito utilizando o framework .NET, fornecido pela Microsoft. Essa plataforma possibilita o desenvolvimento de uma aplicação que pode ser executada em qualquer dispositivo que possua o framework da plataforma. Esse ambiente possui uma interface gráfica que facilita a criação de janelas, botões e textos na aplicação em desenvolvimento. O código foi escrito utilizando a linguagem $\mathrm{C \#}$, pois ela possui maior facilidade de utilização de recursos necessários ao desenvolvimento da aplicação, o que torna o processo mais rápido e simples.

A tela principal do software de controle desenvolvido é apresentada na Figura 4. Essa tela possui um campo "Ar condicionado" que, quando acionado, exibe uma lista com os ambientes cadastrados no arquivo de configuração que podem ser controlados. Apesar de terem sido cadastrados apenas dois ambientes no software de controle, vários aparelhos foram testados em outros ambientes. Após a seleção de um ambiente, informações como o estado (ligado ou desligado) e temperatura são exibidas, desde é claro se o hardware estiver devidamente configurado e conectado a internet. $\mathrm{O}$ aplicativo possui dois botões: um para ligar e outro para desligar o aparelho, sendo que se o aparelho estiver ligado o botão Ligar fica bloqueado e somente é possível ativar o botão Desligar. Entretanto, se o aparelho estiver desligado o botão Desligar fica bloqueado e somente é possível ativar o botão Ligar. 


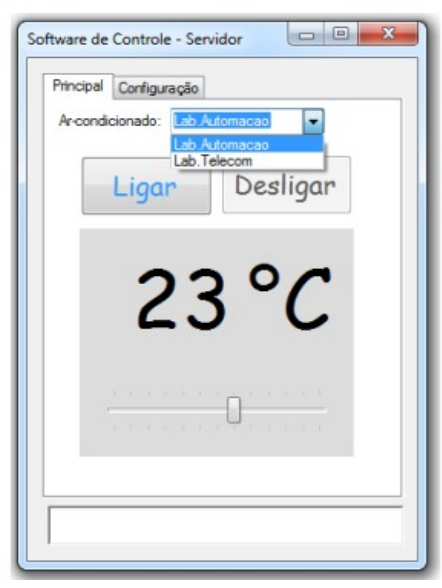

(a)

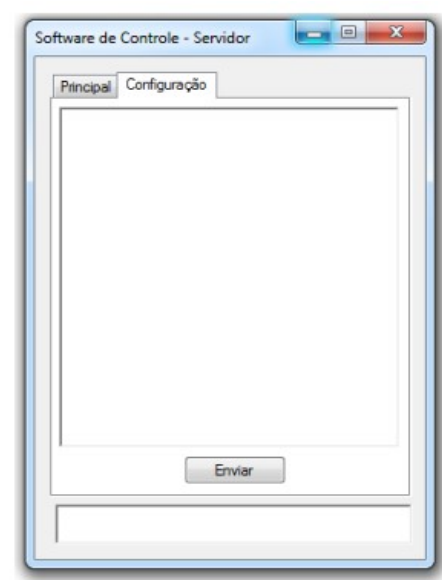

(b)

Figura 4: Telas do software de controle. a) Tela principal; b) Tela de configuração.

\section{Testes e Resultados}

Para validar o dispositivo e verificar seu perfeito funcionamento, foram feitos testes do hardware, do software embarcado e do envio e recebimento de dados pelo sistema todo (dispositivo). O teste de hardware consistiu em verificar o funcionamento de cada componente bem como a continuidade das ligações, também foram testados o nível de tensão em cada componente. No teste do software embarcado, os principais elementos verificados foram a comunicação entre o microcontrolador ATmega328p e o módulo ESP8266-01 e o envio de dados utilizando o LED infravermelho. Para a realização dessa análise, foi utilizado um analisador lógico de 8 canais produzido pela Saleae. O teste da comunicação serial (UART) e o o teste do envio de dados por meio do LED infravermelho foram realizados conectando os pinos (RX e TX) do módulo ESP8266-01 ao analisador lógico, como mostra a Figura 5a. O dispositivo foi alimentado e foi feita uma simulação para envio de dados por infravermelho. Assim, o analisador lógico armazenou os sinais emitidos, que em seguida foram analisados no computador.

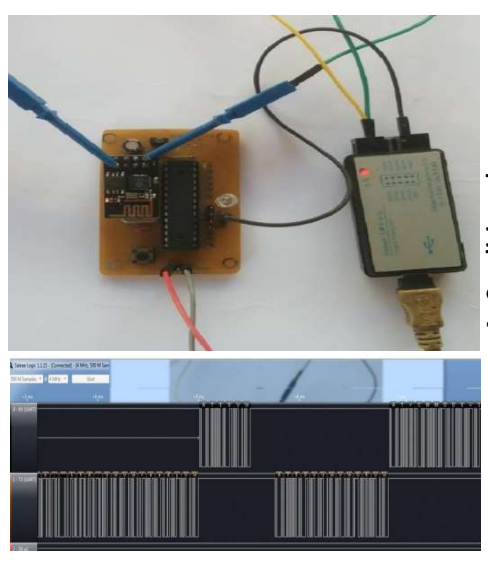

(a)

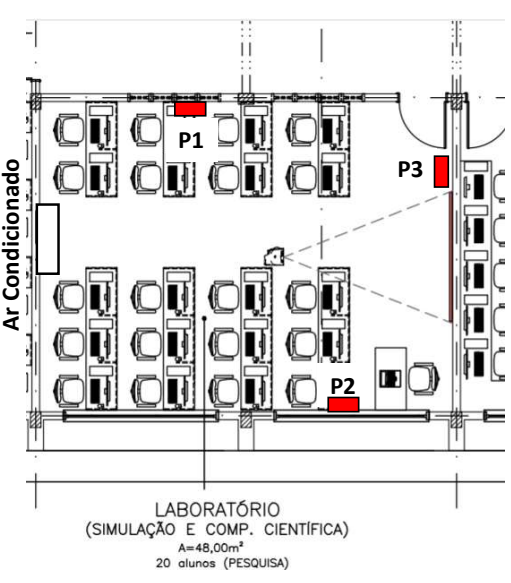

(b)

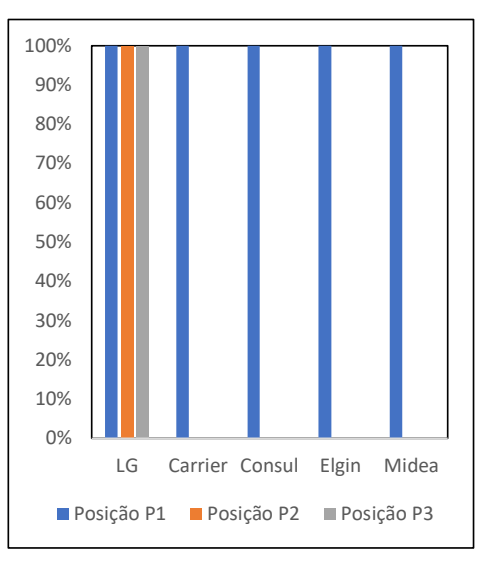

(c)

Figura 5: Telas do software de controle. a) Tela principal; b) Tela de configuração. 
O software de controle desenvolvido foi instalado em um computador conectado a rede da universidade. $\mathrm{O}$ hardware foi ligado a uma fonte de $7,5 \mathrm{~V}$ e configurado de acordo com cada marca de ar condicionado que foi testado e também para se conectar a rede local. O software indentifica o hardware conectado a rede e os testes das funcionalidades foram executados. Apesar do software ter apenas as duas entradas dos laboratórios de Automação e Telecomunicações, o dispositivo foi instalado em diversas outras salas da Universidade Estadual do Maranhão (UEMA) que tinham ar condicionados de fabricantes diferentes, com o objetivo de verificar o funcionamento com as diversas marcas existentes no mercado. As funcionalidades de ligar, desligar, inserir a temperatura desejada e o uso do timer foram testadas em ar condicionados das marcas Carrier, Consul, Elgin, LG e Midea. Cada funcionalidade foi testada 15 vezes. No Laboratório de Computação Científica o hardware foi colocado em 3 posições diferentes em relação a posição do ar condicionado (Figura 5b) e os mesmos testes foram executados. Os testes tiveram $100 \%$ de êxito e nenhum problema nem de software ou de hardware foram detectados durantes os testes realizados (Figura 5c).

\section{Conclusões}

Os benefícios potenciais da Internet das Coisas (IoT) são muitos e estão mudando a maneira que trabalhamos e vivemos, abrindo novas oportunidades para o crescimento da inovação e da criação do conhecimento. Neste trabalho aplicamos os conceitos de IoT para resolver um problema comum em ambientes que possuam muitos equipamentos de ar condicionado para serem gerenciados.

No caso do controle manual e no desligamento in loco dos aparelhos, pode haver falha humana nesse controle, fazendo com que aparelhos de ar condicionado fiquem ligados por longos períodos de tempo desnecessariamente, aumentando os custos com energia elétrica e a frequência da manutenção dos equipamentos e ainda reduzindo o tempo de vida útil do aparelho.

Com a solução proposta e desenvolvida neste trabalho de pesquisa, utilizando um microcontrolador ATmega328p e o módulo ESP8266-01, é possível controlar remotamente todo um sistema de climatização que possua um ou vários equipamentos, em um mesmo local ou em locais separados. Obviamente que para cada aparelho a ser controlado, será necessário um dispositivo. Os testes com o protótipo mostraram sua capacidade de se comunicar com aparelhos de ar condicionado de diversas marcas, sendo apenas necessário que as configurações sejam realizadas corretamente. $O$ software de controle desenvolvido até aqui é muito simples, mas com capacidade de controlar um número significativo de equipamentos remotamente, basta que para isso sejam cadastrados os ambientes no software de controle e identificados corretamente os aparelhos de cada ambiente. Esse software está sendo aprimorado e o objetivo final é uma plataforma de supervisão acessível de computadores e quaisquer dispositivos móveis para gerenciamento das principais funcionalidades já mencionadas no trabalho.

Esse projeto está ainda alinhado com os conceitos de Smart Buildings e de Smart Energy propiciando a aplicação responsável de eficiência energética em todos os tipos de construção, podendo ser aplicado na esfera residencial, empresarial e industrial e contribuindo para o meio ambiente e sustentabilidade global. 


\section{Agradecimentos}

Agradecemos a Fundação de Amparo à Pesquisa do Estado do Maranhão (FAPEMA), agência de fomento que apoiou integralmente o desenvolvimento deste trabalho através dos processos TIAC-06554/16 e UNIVERSAL-01515/16.

\section{Referências}

Diego Rodrigues, Uso de Dados de Mídias Sociais para Desenvolvimento de Metodologia de Posicionamento de Táxis. Simpósio Brasileiro de Redes de Computadores e Sistemas Distribuídos, 2018, Campos do Jordão, Brasil.

Cisco 2019 "Visual Network Index", https://www.cisco.com/c/m/en_us/solutions/ service-provider/visual-networking-index.html, Dezembro, 2019.

Yan, H.; Xia, Y.; Xu, X.; Deng, S.: Inherent operational characteristics aided fuzzy logic controller for a variable speed direct expansion air conditioning system for simultaneous indoor air temperature and humidity control. Energy Build. 158, 558568 (2018).

Omer, S.A.U.R.; Muhammad, E.: Design of intelligent air conditioner controller using fuzzy logic. In: ICIEECT 2017-International Conference on Innovations in Electrical Engineering and Computational Technologies 2017, Proceedings, pp. 1-5 (2017).

Huang, Y.; Khajepour, A.; Ding, H.; Bagheri, F.; Bahrami, M.: An energy-saving setpoint optimizer with a sliding mode controller for automotive airconditioning/refrigeration systems. Appl. Energy 188, 576-585 (2017).

J. Guo, J. Zhou and T. Cui, "Investigation of evaluating and decreasing the energy usage of air conditioning system in supermarket," Refrigeration, Air-conditioning, Electric Power and Mechanism, vol. 26, no. 2, pp. 31-33, 25, February 2005.

Ibrahim, Dragan. "The Complete ESP32 Projects Guide”. 1a. ed.: Elektor Digital, 2017.

NATIONAL COMPUTATION INFRASTRUCTURE. Serial Port I/O. Disponível em $<$ https://nf.nci.org.au/facilities/software/Matlab/techdoc/matlab_external/ch_seri8.ht ml, acessado em Novembro de 2019.

Kolban, N. "Kolban's Book on ESP8266”. 2016. Disponível em: $<$ https://leanpub.com/ESP8266_ESP32>.

Schwartz, M. “Open Home Automation”. 2015. Disponível em: $<$ https://openhomeautomation.net/control-a-lamp-remotely-using-the-esp8266-wifichip/>.

Microchip, “Atmel Studio 7 User Guide”. Microchip Technology Incorporated, 2018, U.S.A. 